\title{
Study of Axial Dimension of Static Head-to-Head Domain Boundary in Amorphous Glass-Coated Microwire
}

\author{
M. Kladivová*, J. Ziman, J. Kecer, P. Duranka \\ Department of Physics, Technical University of Košice, Park Komenského 2, 04200 Košice, Slovakia
}

\begin{abstract}
A new experiment allowing the study of static domain boundary dimensions in bistable microwire is proposed. In this experiment a static domain boundary is created by an inhomogeneous axial magnetic field. The changes in axial magnetic flux due to the presence of this boundary were measured along the microwire using two pick-up coils. Experimental results were compared with a simple theoretical model. For $\mathrm{Fe}_{77.5} \mathrm{Si}_{7.5} \mathrm{~B}_{15}$ microwire with total diameter of $30 \mu \mathrm{m}$ and metal nucleus diameter of $15 \mu \mathrm{m}$, good agreement between theoretical and experimental data was obtained for an axial dimension of the static domain boundary about 200 times larger than the diameter of the wire metallic core.
\end{abstract}

DOI: 10.12693/APhysPolA.131.639

PACS/topics: $75.50 . \mathrm{Kj}, 75.60 . \mathrm{Ch}$

\section{Introduction}

Fe-based glass-coated amorphous ferromagnetic microwires are attractive soft magnetic materials which are used in various technical applications [1,2]. The single domain wall magnetization reversal process which is typical for this group of magnetic materials makes these materials also very suitable for the study of a single domain wall processes as well as domain wall structure. The dynamics of a single domain wall in so-called bistable microwire has been the subject of many studies [3]. However, many questions about the structure and dimension of this type of domain wall still remain open. Most authors dealing with this subject deduce the shape and dimension of this wall from the analysis of voltage peaks induced in the Sixtus-Tonks experiments $[4,5]$. The information obtained from this analysis is about a moving wall. The properties of the pick-up coil and stray field around this type of domain boundary have to be taken into account for correct interpretation of the results obtained in this way.

Present work deals with the study of static domain boundary dimensions. A simple theoretical model in which the stray field is also included enables estimation of the domain wall length.

\section{Experiment}

The experimental setup is depicted in Fig. 1. It consists of two magnetizing coils (solenoids So1, So2) and two coaxial pick-up coils (PuC1, PuC2). Solenoids So1 and So2 have the same geometry (diameter $D=3 \mathrm{~cm}$, length $l=15 \mathrm{~cm}$ ) and equal number of turns (1000). They have a common axis and are in contact by their ends (see Fig. 1). The solenoids are connected in series through switches to the power supply so that equal current flows through the windings of both solenoids.

\footnotetext{
*corresponding author; e-mail: maria.kladivova@tuke.sk
}

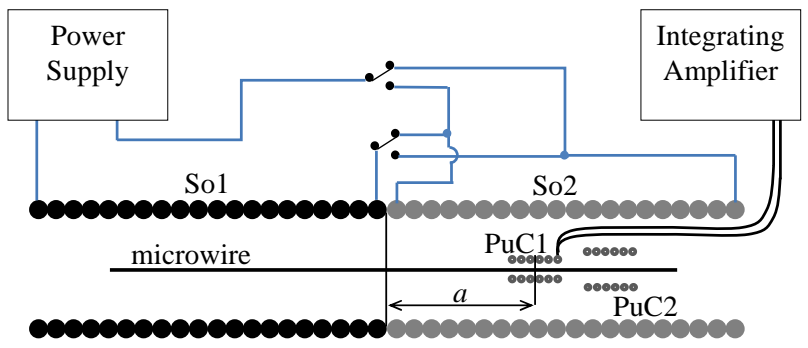

Fig. 1. Experimental setup.

The switches allow solenoids So1 and So2 to be connected in such a way that they create fields in the same or in opposite directions. In the first case the situation is the same as for a standard solenoid of length $2 l$. If the fields created by solenoids So1 and So2 have opposite direction and appropriate magnitude, a single domain wall can be created in bistable microwire. The position of this wall is close to the place where the sum of the fields created by solenoids So1 and So2 is equal to zero. The microwire is placed in two pick-up coils. Each pick-up coil can be connected to the input of an integrating amplifier so that magnetic flux through its winding can be measured [6]. Pick-up coils $\mathrm{PuC} 1$ and $\mathrm{PuC} 2$ have equal length and number of turns $(5 \mathrm{~mm}, 287)$, but they differ in the average radius of turns ("small" $R_{1}=0.34 \mathrm{~mm}$, "large" $R_{2}=0.85 \mathrm{~mm}$ ). The position of the microwire is fixed in the magnetizing coils, but the pick-up coils can be moved along the microwire. Let a particular pick-up coil be located at a given position $a$. The measuring procedure consists of two steps. In the first, solenoids So1 and So2 are connected in such a way that current through them, which is switched on for about one second, creates opposite fields. A stable single domain boundary between axial domains is created by these fields [7]. In the second step, solenoids So1 and So2 are connected in such a way that they create fields with the same direction, and the magnetic flux in pick-up coil is measured [6]. Measurements were performed on an as-cast amorphous 
ferromagnetic glass-coated $\mathrm{Fe}_{77.5} \mathrm{Si}_{7.5} \mathrm{~B}_{15}$ microwire with metallic nucleus diameter of about $15 \mu \mathrm{m}$ and glass layer thickness of about $7.5 \mu \mathrm{m}$; sample length was $12 \mathrm{~cm}$. Experimental dependences of normalized magnetic flux $\Psi=\Phi / \Phi_{0}\left(\Phi_{0}\right.$ is magnetic flux measured far from the wall) vs. wall position obtained in this way are depicted in Fig. 2.

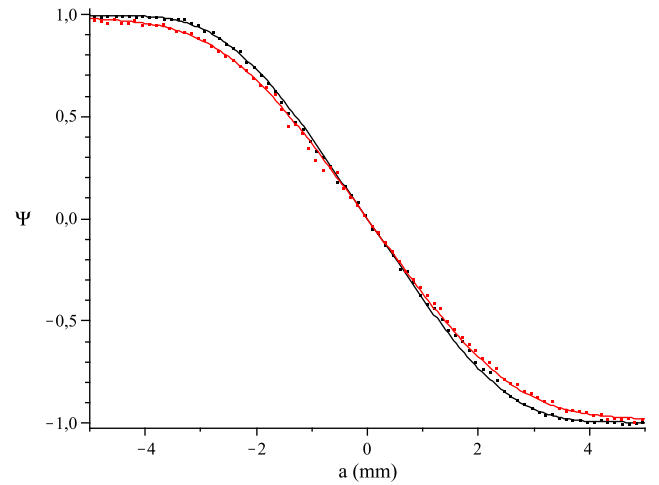

Fig. 2. Experimental data for pick-up coils with "small" (black points) and "large" (red points) radius with corresponding model curves for the wall length $L=3 \mathrm{~mm}$, see Figs. 5, 6 .

\section{Results and discussion}

A simple model of planar DW (2D object) as depicted in Fig. 3 was used for the theoretical calculations of magnetic flux vs. pick-up position with wall length as a parameter.

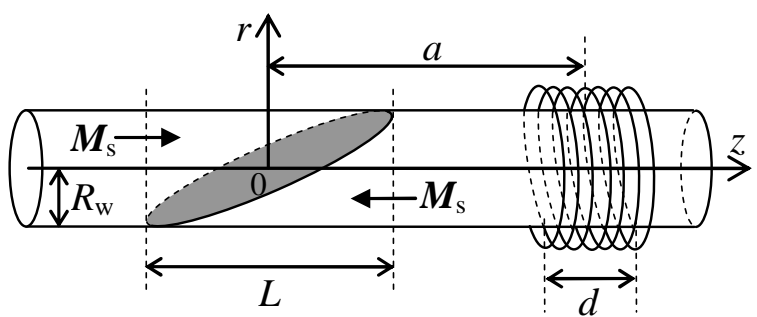

Fig. 3. Model of planar head-to-head wall, $L$ - wall length, $R_{w}$ - wire radius, $\boldsymbol{M}_{s}$ - magnetization, $d-$ coil length, $a$ - distance between wall centre and pickup coil centre.

Comparison of experimental with theoretical data produced the possibility of estimating the characteristic wall length $L$ (width). It should be noted that the pick-up coil signal reflects not only magnetic flux due to magnetization of domains in the microwire (i.e. the wall length), but also any stray field of magnetic charge accumulated near the wall. Thus there are two contributions to the magnetic flux $\Phi$ through the winding of the pick-up coil

$$
\Phi=\Phi_{w}+\Phi_{d}
$$

where $\Phi_{w}$ is axial magnetic flux due to magnetization inside domains and $\Phi_{d}$ due to the stray field.

First the magnetic flux $\Phi_{w 1}$ across the wire crosssection (see Fig. 4) is obtained as a function of distance

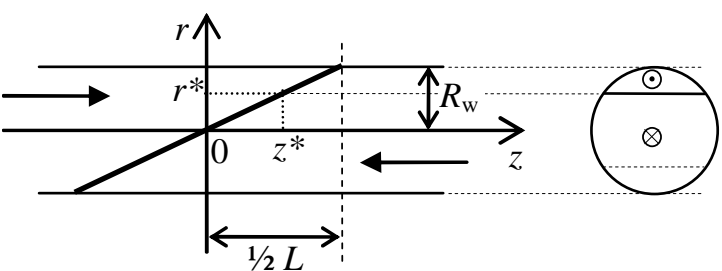

Fig. 4. Side and cross view of magnetization in the wire.

$z$ of a given wire cross-section from the wall centre. For $z \in\langle-L / 2, L / 2\rangle$ :

$$
\Phi_{w 1}=-\frac{\Phi_{0}}{n} \frac{2}{\pi}\left[\frac{2 z}{L} \sqrt{1-\left(\frac{2 z}{L}\right)^{2}}+\arcsin \left(\frac{2 z}{L}\right)\right],
$$

where $\Phi_{0}=n \mu_{0} M_{s} \pi R_{w}^{2}$ and $n$ is the number of turns in the coil. For $z<-L / 2$ and $z>L / 2$ the flux $\Phi_{w 1}$ is $\Phi_{0} / n$ and $-\Phi_{0} / n$, respectively. The total flux $\Phi_{w}$ in a coil of length $d$ whose centre is at distance $a$ from the wall centre can now be obtained by summing the flux for all $n$ turns (i.e. for $z \in\langle a-d / 2, a+d / 2\rangle$ ). For high density of turns $n / d$ of pick-up coils used in this experiment summation can be replaced with integration

$$
\Phi_{w}=\frac{n}{d} \int_{a-\frac{d}{2}}^{a+\frac{d}{2}} \Phi_{w 1}(z) \mathrm{d} z
$$

Since the integral of the function in square brackets in Eq. (2) is known, the magnetic flux $\Phi_{w}$ as a function of $a$ can be calculated.

A simple model was used for calculating the magnetic flux of stray field $\Phi_{d}$. First the axial component of the stray field was obtained. Magnetic charge $Q_{m}=2 M_{s} \pi R_{w}^{2}$ was modelled as symmetrically distributed with respect to the wall centre along the $z$ axis. The radii of both pick-up coils were quite large $\left(R_{1,2} \ll R_{w}\right)$, so the charge could be considered as distributed over a one-dimensional object. Density of charge on the wall is assumed to be constant, so the part of charge $\mathrm{d} Q_{m}$ at length $\mathrm{d} z$ is proportional to the corresponding area of the wall $\mathrm{d} S$. The projection of the wall area in direction $z$ is an ellipse with semi-axes $L / 2$ and $R_{w}$. Obtained $\mathrm{d} Q_{m}$ at position $z$ is

$$
\mathrm{d} Q_{m}=\frac{2 Q_{m}}{\pi R_{w} L} \mathrm{~d} S=\frac{4 Q_{m}}{\pi L} \sqrt{1-\left(\frac{2 z}{L}\right)^{2}}
$$

and the corresponding axial component of the stray field at distance $b$ from the wall centre at point $[b, r]$ is

$$
\mathrm{d} H_{d}(b, r)=\frac{\mathrm{d} Q_{m}}{4 \pi} \frac{b-z}{\left[(b-z)^{2}+r^{2}\right]^{\frac{3}{2}}} .
$$

Magnetic flux $\mathrm{d} \Phi_{d 1}$ caused by $\mathrm{d} Q_{m}$ at position $z$ across one coil turn with radius $R$ at distance $b$ from the wall centre can be obtained by integration of $\mu_{0} \mathrm{~d} H_{d}(b, r)$ (Eq. (5)) over the area of the turn 


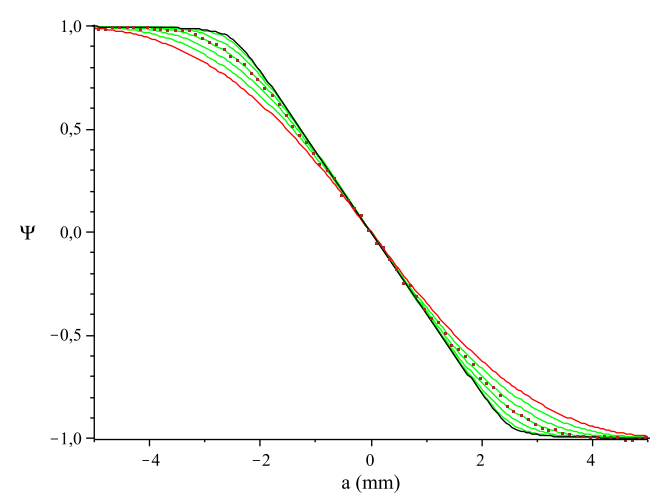

Fig. 5. Model curves for the "small" pick-up coil for $L=0$ (black line), $L=1,2,3,4,5 \mathrm{~mm}$ (green lines) and for $L=6 \mathrm{~mm}$ (red line); dots are measured experimental data.

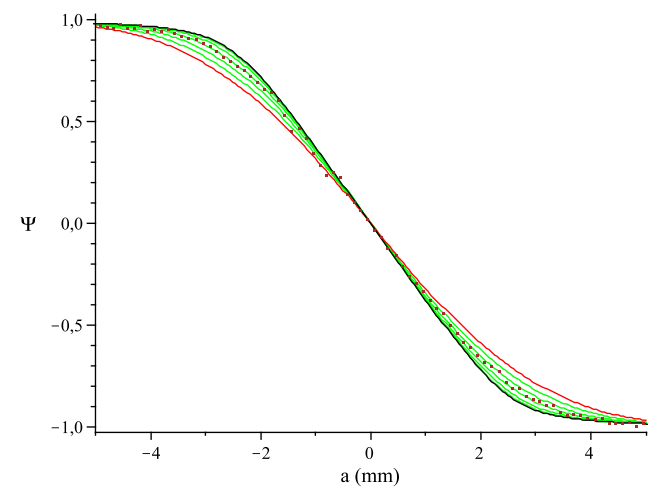

Fig. 6. Model and experimental dependences for the "large" pick-up coil.

$$
\mathrm{d} \Phi_{d 1}=\int_{0}^{R}\left[\mu_{0} \mathrm{~d} H_{d}(b, r) 2 \pi r\right] \mathrm{d} r
$$

Total $\Phi_{d 1}$ from $Q_{m}$ over one turn at distance $b$ from the wall centre is

$$
\begin{gathered}
\Phi_{d 1}=\int_{(L)} \mathrm{d} \Phi_{d 1}=\frac{\Phi_{0}}{n} \frac{4}{\pi L} \int_{-\frac{L}{2}}^{\frac{L}{2}} \sqrt{1-\left(\frac{2 z}{L}\right)^{2}} \\
\times\left[\frac{b-z}{\sqrt{(b-z)^{2}}}-\frac{b-z}{\sqrt{(b-z)^{2}+R^{2}}}\right] \mathrm{d} z .
\end{gathered}
$$

(The integral in Eq. (7) can be expressed via elliptic functions.) Total flux over the whole pick-up coil can now be obtained by integration of fluxes over all pick-up coil turns

$$
\Phi_{d}=\frac{n}{d} \int_{a-\frac{d}{2}}^{a+\frac{d}{2}} \Phi_{d 1}(b) \mathrm{d} b,
$$

( $b \equiv z$ in Fig. 3). Integration was performed numerically.
Total flux over the pick-up coil obtained using Eq. (1) and Eqs. $(3,7)$ depends on distance $a$ with wall length $L$ as a parameter. The obtained theoretical and experimental dependences for normalized axial magnetic flux $\Psi$ vs. distance $a$ of the pick-up coil from the wall centre are depicted in Figs. 5 and 6.

It can be seen that the stray field influences the measured magnetic flux in the pick-up coil. For both pick-up coils the best agreement was obtained for domain wall length $L=3 \mathrm{~mm}$ (see Fig. 2), whereby this length is about 200 times larger than the diameter of the wire metallic core.

\section{Conclusions}

The experimental and theoretical results presented in this paper confirm that the stray field has to be taken into account when information about domain wall dimension is deduced from analysis of the signal induced in pick-up coil. For the microwire used in our experiment, the static domain wall is symmetrical with respect to its centre and domain wall length is about 200 times larger than the diameter of the wire metallic core.

\section{Acknowledgments}

This research was supported by the project "Centre of Excellence for Integrated Research \&Exploitation of Advanced Materials and Technologies in Automotive Electronics" ITMS 26220120055 and also by VEGA grant No. 1/0413/15 of the Scientific Grant Agency of the Ministry for Education of the Slovak Republic.

\section{References}

[1] V. Zhukova, M. Ipatov, A. Zhukov, Sensors 9, 9216 (2009).

[2] M. Vázquez, H. Chiriac, A. Zhukov, L. Panina, Phys. Status Solidi A 208, 493 (2011).

[3] M. Vázquez, Handbook of Magnetism and Advanced Magnetic Materials, Vol. 4, 2007, p. 1.

[4] H. García-Miquel, D.-X. Chen, M. Vázquez, J. Magn. Magn. Mater. 212, 101 (2000).

[5] S.A. Gudoshnikov, Yu.B. Grebenshchikov, B.Ya. Ljubimov, P.S. Palvanov, N.A. Usov, M. Ipatov, A. Zhukov, J. Gonzalez, Phys. Status Solidi A 206, 613 (2009).

[6] J. Ziman, B. Zagyi, J. Magn. Magn. Mater. 169, 98 (1997).

[7] J. Ziman, J. Onufer, M. Kladivová, J. Magn. Magn. Mater. 323, 3098 (2011). 